\title{
The Meta-Analysis in Medicine: Tools for Clinical and Experimental Research
}

\author{
Daniel Cadena Sandoval ${ }^{1}$ and Carlos Alfonso Tovilla Zarate ${ }^{1,2 *}$
}

${ }^{1}$ Universidad Juárez Autónoma de Tabasco, División Académica Multidisciplinaria de Comalcalco, Comalcalco, Tabasco, México ${ }^{2}$ CIGEN, centro de investigación genómica, Comalcalco Tabasco, México

\begin{abstract}
The meta-analysis is a tool used to know results of previous research and allows obtaining a result globally. It is known that increase the statistics power and have an estimate of the treatment effect. It allows us to combine the results of studies with different results. Therefore in this paper we present some generalities about the development and the main points to consider when performing a meta-analysis. Meta-analysis is used in different fields of knowledge including genome wide genetic associations, so it is necessary for evidence-based medicine.
\end{abstract}

\section{Introduction}

The number of scientific publications have experienced in recent years a remarkable growth which suggests that they have exceeded the capacity of scientific quality controls systems. There are data that around 2,500 new publications are indexed annually on Medline [1]. The scientific evidence is not the result of one single effort but of integration and replication of results from different studies, and it is common to find studies with the same objectives that have a little homogeneous results or even contradictory. The systematic reviews make explicit all decisions taken in the revision process (selection criteria of the original papers, methods to combine the results and so on) also, systematize the process with the aim to obtain more exhaustive reviews and reduce the possibilities of bias in results and their interpretations.

The classical approaches to summarize the evidence include narrative reviews, systematic reviews and meta-analysis, and the use of the last one has a prominent role in the validation and interpretation of the results of clinical studies [2]. The limitations of this type of review is the possibility of incurring bias arising (a) of the original studies, (b) the investigator-subjective caused by the inclusion of studies, (c) of the poor quality of studies, or (d) of a misinterpretation of the response. These limitations have provoked apparition of systematic reviews to perform a synthesis of the scientific evidence.

\section{Definition}

The term meta-analysis was coined in 1976; although, the first metaanalysis is attributed to Pearson (1904), who analyzed data from five studies on the correlation between the vaccination for enteritic fever and its mortality. This identifies a process of analysis retrospectively performed on available published data on a specific topic [2].

The meta-analyses are powerful tools used to synthesize the available evidence in any field of research, medicine, ecology, social science and others; they can provide evidence for policy makers to judge risks, benefits, and harms of health care behaviors and interventions. Also it is a good starting point for clinical practice guideline developers; provide summaries of previous research for funders wishing to support new research; and help editors judge the merits of publishing reports of new studies [3]. In recent years the applications of meta-analysis are not confined exclusively to clinical trials. It has been fruitful in several other interesting fields likewise (e.g., observational studies, dose-response, or studies evaluating diagnostic test). Indeed, randomized clinical trials provide more useful evidence than that offered the cohort, and these better than that provided by case-control studies. However, in many situations randomized controlled designs are not feasible and only data from observational studies are available [4]. A correct systematic review on a topic requires collection and analysis of all published data and not only of those which are more interesting, relevant or easily available. Two steps are thus important for the analysis: first, a complete collection of the published literature, and second, the synthesis of the information acquired [5].

Since its inception in the field of medicine, meta-analysis has been used mainly for combining results of randomized clinical trials to ensure that medical treatments are based on the best available empirical data. The revisions updated continuously, such as those offered by the Cochrane Database of Systematic Reviews facilitate performing the meta-analysis in this type of experimental studies [6]. The same form, the Preferred Reporting Items for Systematic Reviews and Meta-Analyses (PRISMA). A consensus process that was informed by evidence, whenever possible, was used to develop a 27-item checklist; items deemed essential for transparent reporting of a systematic review were included in the checklist. Also, was developed a four-phases flow diagram, modified from the originally proposed by QUOROM [7].The PRISMA Statement itself provides further details regarding its background and development [3]. PRISMA focuses on ways in which authors can ensure the transparent and complete reporting of systematic reviews and meta-analyses. It does not address directly or in a detailed manner the conduct of systematic reviews, for which other guides are available [8].

\section{Aims}

One of the main objectives of clinical research is to obtain clear and reliable results that can be utilized in the management of patients and

*Corresponding author: Carlos Alfonso Tovilla Zárate, División Académica Multidisciplinaria de Comalcalco, Ranchería Sur, Cuarta Sección, C.P. 86650 Comalcalco, Tabasco, México, Tel: 529933581500 ext. 6900; E-mail: alfonso_ tovillaz@yahoo.com.mx

Received March 20, 2012; Accepted April 27, 2012; Published April 30, 2012

Citation: Sandoval DC, Tovilla Zarate CA (2012) The Meta-Analysis in Medicine: Tools for Clinical and Experimental Research. J Biomet Biostat S7:011. doi:10.4172/2155-6180.S7-011

Copyright: (C) 2012 Sandoval DC, et al. This is an open-access article distributed under the terms of the Creative Commons Attribution License, which permits unrestricted use, distribution, and reproduction in any medium, provided the original author and source are credited. 
possibly as a basis for clinical guidelines. Clinical trials do not always reach this target and often give contrasting results. Meta-analysis, if correctly used, contributes to achieve this target, and allows for a critical evaluation of the studies under consideration $[9,10]$.

The objectives, in any case must be raised very clearly: 1) Test the hypothesis related to the effect (in particular, the direction) of the intervention under study; 2) Increase the accuracy of the estimators of the effect (in particular, their magnitude) of the intervention under study; 3) To assess the consistency between clinical trials of similar interventions associated with the topic and generate a more efficient estimator of effect; 4) To assess the consistency between trials of different interventions performed for the same purpose and generate an estimate of the effect of such care; 5) Identify with accuracy subgroups of patients who would most likely be affected by the intervention, either in a favorable or unfavorable manner. 6) Calculate the requirements in terms of sample size, of future clinical trials to perform in the same field [2].

In addition, the meta-analysis contributes to many aspects: 1) Increases the statistical power of a comparison; 2) Improves the estimation of the effect of a treatment; 3) Combines the results of studies that are contrasting; 4) Answers new questions; 5) Analyses subgroups of subjects selected from different studies. 6) Analyzes trends (e.g. within a time-frame, in a sub-group of patients with the same characteristics). 7) Defines areas in which further studies are needed. 8) Analyzes if and how previous studies have modified knowledge on a certain topic $[1,2,10]$.

\section{Design of Meta-Analysis}

\section{Quantify effects}

The researcher has to establish measures that will be used to describe and represent the effects, so they can be added. These measures will depend both on the type of response (binary/continuous) and study design (experimental/observational) used in the primary studies $[2,5]$.

Binary response: This type of response has only two categories of outcome (death/survival, sick/healthy, etc.). In studies with this type of response, effect measures most commonly used are the hazard ratio (or relative risk), the odds ratio (odds ratio), or risk difference $[11,12]$.

Continuous response: In the literature there are many studies in which the response variable is measured in continuous scale (systolic blood pressure, cholesterol, etc.). If the answers of all studies are measured on the same scale, then the measure of effect (or effect size, effect size) is simply the difference between group means. If not, that is, if studies measure the response on a different scale, we must consider instead a standardized difference of means.

\section{Search for information}

In general, each of the studies that may be involved in the metaanalysis, want to extract information regarding the following: The study characteristics; such as type of design, characteristics of the study sample (age, gender, prognostic factors, etc.), type of operation (active ingredient, regimen, dose, etc.), outcome measures considered, time tracking and other features for evaluating the degree of homogeneity or heterogeneity of the studies to be combined.

The methodological quality of studies: there are different instruments to assess qualitatively or quantitatively the possible existence of bias and therefore, called internal validity of the original studies $[1,3]$. The results of the studies: measures of effect observed (odds ratio, relative risk, risk difference, mean difference, etc.) with indicators of variability (confidence intervals) and statistical significance.

\section{Locate of the research studies}

5.3.1) Sources of information: To carry out literature searches can be assumed from informal sources, primary sources and secondary sources: 1) Informal sources are comprised of personal files, books, review articles, expert contacts; 2) Primary sources are known journals related to the subject and the realization of upward revisions (from bibliographies of articles already have and so on). 3) Secondary sources are the databases that are critical to the performance of any literature search. The major databases are MEDLINE, EMBASE, Web of Science and Cochrane databases.

Example: The following example from our studies of meta-analysis indicate the application of locate of the research studies.

Identification and selection of publications: A literature search comprising from January to March 2011 was performed. The publications were identified using the following search terms in Medline, PubMed and Web of Science databases: "COMT and suicidal behavior", "COMT and suicide", rs4680 and suicidal behavior", rs4680 and suicide" and "COMT Val/Met and suicide". These words were combined to retrieve the summaries. The search also implicated the review of the bibliography cited at the end of various research articles to identify additional papers not covered by the electronic search of abstracts [9].

\section{Inclusion/exclusion criteria}

In meta-analysis, sampling units are the results and studies published or unpublished. The inclusion/exclusion criteria give a "definition of case" for results that will be used in the synthesis. Here, might be incurring in an important bias, the bias of selection. To reduce the risk of committing it has to ensure that the review of the studies carried out in blinded way or masked (without knowing the journal, year of publication, authors and institutions) developing a list of inclusion and exclusion criteria, which are always objectively analyzable and methodological and not by results $[2,5,10]$.

Example: To be selected, the publications had to fulfill the following criteria: (1) to be published in peer-reviewed journals, (2) to be written in English, (3) to contain independent data, (4) to be casecontrol association studies in which the frequencies of three genotypes were clearly stated or could be calculated, and (5) the use of healthy individuals as controls $[11,12]$.

\section{Evaluation of the quality of the studies included}

There are some basic aspects that must be controlled from the information obtained: study design, the possibility of combining the different studies, bias control, and statistical analysis of each study was successful.The most common use of quality scores of studies is to assign greater weight to some studies than others when combined. Another interesting use of these measures would be to conduct studies groups according to their quality, and determine whether the degree of it relates to the estimates of the effects $[2,10]$.

\section{Analysis of heterogeneity}

All statistical tests designed to verify the existence of heterogeneity are based on the hypothesis that the variability between studies is 
zero. One test is most appropriate to value this; the Q test given by DerSimonian and Laird, preferred by questions of validity and computational simplicity. Despite its advantages, this test has low statistical power, mainly because the number of primary studies that are often considered (i.e., the effective sample size) is generally small. Due to the low power of statistical tests, they can be supplemented with a graphical representation that allows visual inspection of the magnitude of variability between studies, and/or using the parameter tau-squared $\left(\tau^{2}\right)$, which is defined as the variance of the true effect sizes. In other words, if we had an infinitely large sample studies, each, itself, infinitely large (so that the estimate in each study was the true effect) and computed the variance of these effects, this variance would be $\tau^{2}$. Since we cannot observe the true effects we cannot compute this variance directly. Rather, we estimate it from the observed effects, with the denoted $T^{2}$. This method does not make any assumptions about the distribution but to assign study weight under the random effects model [10].

However for observed the real proportion of dispersion is necessary the use of $I^{2}$ index, which is the proportional of observed dispersion that is real, rather than spurious. It is not dependent on the scale, and is expressed as a ratio with a range of $0 \%$ to $100 \%$. For example, if $I^{2}$ is near zero, then almost all the observed variance is spurious, which means that there is nothing to explain. By contrast, if $I^{2}$ is large, then it would make sense to speculate about reasons for the variance, and possibly to apply techniques such as group analysis or meta-regression to try and explain it $[10,13]$.

The Galbraith's plot. It is recommended to be applied to any type of study (observational and experimental). Represents the precision of each study (the inverse of the standard error) against the standardized effect, also represents the fitted regression line to these points and a confidence band. Studies that fall outside this band are the main contributors to heterogeneity. Moreover, the position of studies in the abscissa visually identifies those who have more weight in the metaanalysis. This graph can also be used to detect sources of heterogeneity in labeling studies by different variables, such as year of publication.

The L'Abbé plot. It is more restrictive, applicable only to metaanalysis of clinical trials. It represents the event rate in the treatment group compared to the rate control group. So this graph only occurs when the response variable is binary.

If there is heterogeneity between studies (statistical and/or clinical), it can take several attitudes. Methodologically, the less risky would not proceed with a summary of the primary studies. Finally, if it is suspected that there are reasons that may explain the heterogeneity of results between studies; the recommended option is to perform a subgroup analysis combining only studies that meet certain condition or characteristic, so that they are more homogeneous.

\section{Combination of results}

The choice of method depends mainly on the type of outcome/ effect used and assessing the degree of heterogeneity of study results.

Estimated combined effect, Fixed-effects model, Random-effects model. The heterogeneity between studies may be taken into account in the analysis using random effects models, or may not be included, if using a fixed effects model.

The fixed-effect model: Assumes a unique effect on the population and does not take into account the variability of results between studies.
Thus, the size of the study and its own variance (intra-study variability) are the only determinants of their weight in the meta-analysis $[2,10,13]$.

The random effects model: It takes into account the possible heterogeneity in finding that the effects of exposure / intervention in the population are diverse and that the studies included in the review are only a random sample of all possible effects. A limitation of the random effects models is the assumption that the included studies are representative of a hypothetical population of studies and the heterogeneity between trials can be represented by a single variance. Another disadvantage is that it grants excessive weight to studies with small sample size.

Examples: The following example from our studies of metaanalysis indicates the application of the random effects model.

Statistical analysis: The data from each of the included studies were classified by phenotype (patients with schizophrenia and healthy controls) and the presence or absence of at least one $\varepsilon 4$ allele of ApoE, considered the risk allele. The OR estimates the probability that the risk allele is present more often in cases compared with healthy controls. An OR greater than 1 suggests a positive association of risk allele and schizophrenia. The results of each of the studies included in the meta-analysis were expressed by the random effects model, for which we calculated the OR for each study and the confidence interval set at $95 \%$. The significance of the OR of all studies was determined by the $\mathrm{Z}$ test. They conducted a test of heterogeneity between the studies selected for the purpose of determining whether the effect size from a homogeneous sample $[9,11,12]$.

\section{Interpretation of results}

The interpretation of the results obtained with a meta-analysis is the result of a series of evaluations that start from the evaluation of the size of the pooled effect, the possible causes of heterogeneity, the evaluation of the 'stability' of the meta-analysis.

Graphical representation of results: The graphical representation of the results of a meta-analysis contributes to a quick and easy interpretation. To this end, constructs a graph in which on the abscissa axis (X axis) is depicted as viewed effect (odds ratio, relative risk, etc.), and along the coordinate axis ( $\mathrm{Y}$ axis) lie different studies, usually ordered by publication year or any other sort. For each study and for the overall estimate of effect, it represents the point estimate and confidence interval that corresponds (assuming a fixed effects model and/or random effects).

\section{Limitations}

The meta-analytic techniques have certain limitations of their methodology. First, the meta-analysis can lead to distorted results due to possible selection bias and publication of studies. Furthermore, the validity of the results and conclusions of the meta-analysis depend on the quality of individual studies so that the combination of biased studies can further enhance the bias. Finally, the interpretation of metaanalysis for heterogeneity or variability between studies is difficult and controversial. The obligation of those who use the meta-analysis is to understand these limitations and discuss them explicit and in each case. Now it describes some of the main methodological problems $[2,12]$.

Heterogeneity between studies: A first methodological critique of the meta-analysis is to attempt a statistical combination of results from studies that show great variability among them. This is not a difficulty unique to meta-analysis, since it is shared by all clinical research, where 
Citation: Sandoval DC, Tovilla Zarate CA (2012) The Meta-Analysis in Medicine: Tools for Clinical and Experimental Research. J Biomet Biostat S7:011. doi:10.4172/2155-6180.S7-011

Page 4 of 4

the wide variety of features inherent in the study subjects is necessary to design a uniform protocol, conduct a rigorous process of selection of study subjects and then careful analysis of the influence on the results of the extreme cases [5].

Publication bias: Selective publication of studies based on their findings; present a risk to the validity of any meta-analysis. It is well known that many research works are completed but not get published. This is more common when the test outcome is "negative"; it is when not demonstrate significant differences between the groups compared or when it is unfavorable to a new drug. For that reasons, meta-analysis that only includes published studies about the same objective in study tend to show biased results. By its nature, it is very difficult avoid the publication bias $[2,9,11,12]$.

\section{Conclusion}

Actually the meta-analysis gives a summarizing of dates in different areas. The meta-analysis enlarges the questions and answers in areas such as diagnostic, prognostic and recently of genetic association. However quality need to be done keeping in mind the requirements for it and understand each of the component parts of the meta-analysis. Today, the meta-analysis becomes relevant because they are essential for evidence-based medicine.

\section{Acknowledgements}

The authors gratefully acknowledge to reviewers for your contributions to the improvement manuscript.

\section{References}

1. Moher D, Tetzlaff J, Tricco AC, Sampson M, Altman DG (2007) Epidemiology and reporting characteristics of systematic reviews. PLoS medicine 4: e78.

2. Leandro G (2005) Meta-analysis in Medical Research: A Handbook for the
Understanding and Practice of Meta-analysis Milan, Italy: Blackwell Publishing

3. Liberati A, Altman DG, Tetzlaff J, Mulrow C, Gotzsche PC, et al. (2009) The PRISMA statement for reporting systematic reviews and meta-analyses of studies that evaluate health care interventions: explanation and elaboration. PLoS Med 6: e1000100.

4. Stroup DF, Berlin JA, Morton SC, Olkin I, Williamson GD, et al. (2000) Metaanalysis of observational studies in epidemiology: a proposal for reporting Meta-analysis Of Observational Studies in Epidemiology (MOOSE) group. JAMA : the journal of the American Medical Association 283: 2008-2012.

5. Nordmann A, Kasenda B, Briel M (2012) Meta-analyses: what they can and cannot do. Swiss Med Wkly 142: w13518.

6. Chalmers I, Dickersin K, Chalmers TC (1992) Getting to grips with Archie Cochrane's agenda. BMJ 305: 786-788.

7. Moher D, Cook DJ, Eastwood S, Olkin I, Rennie D, et al. (1999) Improving the quality of reports of meta-analyses of randomised controlled trials: the QUOROM statement. Quality of Reporting of Meta-analyses. Lancet 354: 1896-1900.

8. Swartz MK (2011) The PRISMA statement: a guideline for systematic reviews and meta-analyses 25: 1-2.

9. Tovilla-Zarate C, Juarez-Rojop I, Ramon-Frias T, Villar-Soto M, Pool-Garcia $S$, et al. (2011) No association between COMT val158met polymorphism and suicidal behavior: meta-analysis and new data. BMC psychiatry 11: 151.

10. Borenstein M, Hedges LV, Higgins JPT, Rothstein HR (2009) Introduction to Meta-analysis UK: John Wiley and Son.

11. Tovilla-Zarate C, Camarena B, Apiquian R, Nicolini H (2008) [Association study and meta-analysis of the apolipoprotein gene and schizophrenia]. Gac Med Mex 144: 79-83.

12. Angles MR, Ocana DB, Medellin BC, Tovilla-Zarate C (2012) No association between the HTR1A gene and suicidal behavior: a meta-analysis. Rev Bras Psiquiatr 34: 38-42.

13. Higgins JP, Thompson SG, Deeks JJ, Altman DG (2003) Measuring inconsistency in meta-analyses. BMJ 327: 557-560.
This article was originally published in a special issue, Medical statistics Clinical and experimental research handled by Editor(s). Dr. Herbert Pang Duke University, USA. 